\title{
Cost-Effectiveness of Peer-Delivered HIV Self-Tests for MSM in Uganda
}

\author{
Stephen Okoboi ${ }^{1,2,3 *}$, Barbara Castelnuovo ${ }^{1}$, Jean-Pierre Van Geertruyden ${ }^{2}$, \\ Oucul Lazarus ${ }^{4}$, Lung Vu ${ }^{5}$, Sam Kalibala ${ }^{5}$, Yvonne Kamara ${ }^{1}$, Perez N. Ochanda ${ }^{1}$, \\ Rachel King ${ }^{6,7}$ and Andrew Mujugira ${ }^{1,77}$
}

${ }^{1}$ College of Health Sciences, Infectious Diseases Institute, Makerere University, Kampala, Uganda, ${ }^{2}$ Faculty of Medicine and Health Sciences and Global Health Institute, University of Antwerp, Antwerp, Belgium, ${ }^{3}$ Global Health Institute, Clarke International University, Kampala, Uganda, ${ }^{4}$ The AIDS Support Organization (TASO), Kampala, Uganda, ${ }^{5}$ Population Council, Washington, DC, United States, ${ }^{6}$ Department of Global Health, University of California, San Francisco, San Francisco, CA, United States, ${ }^{7}$ School of Public Health, Makerere University, Kampala, Uganda

OPEN ACCESS

Edited by:

Donaldson Fadael Conserve George Washington University,

United States

Reviewed by:

Weiming Tang,

University of North Carolina at

Chapel Hill, United States

Liliane Okdah,

King Abdullah International Medical

Research Center (KAIMRC),

Saudi Arabia

*Correspondence:

Stephen Okobo

okoboi25@gmail.com;

sokoboi@idi.co.ug

tThese authors have contributed equally to this work

Specialty section:

This article was submitted to Infectious Diseases - Surveillance,

Prevention and Treatment,

a section of the journal

Frontiers in Public Health

Received: 09 January 2021

Accepted: 23 February 2021

Published: 17 March 2021

Citation:

Okoboi S, Castelnuovo B, Van Geertruyden J-P, Lazarus O, Vu L, Kalibala S, Kamara Y, Ochanda PN

King $R$ and Mujugira A (2021) Cost-Effectiveness of Peer-Delivered HIV Self-Tests for MSM in Uganda.

Front. Public Health 9:651325.

doi: 10.3389/fpubh.2021.651325
Introduction: Distribution of HIV self-testing (HIVST) kits through MSM peer networks is a novel and effective strategy to increase HIV testing coverage in this high-risk population. No study has evaluated the cost or cost effectiveness of peer distribution of HIVST strategies among MSM in sub-Saharan Africa.

Methods: From June to August 2018, we conducted a pilot study of secondary MSM peer HIVST kit distribution at The AIDS Support Organization at Entebbe and Masaka. We used an ingredients approach to estimate the cost of MSM peer HIVST kit distribution relative to standard-of-care (SOC) hotspot testing using programme expenditure data reported in US dollars. The provider perspective was used to estimate incremental cost-effective ratios per HIV infection averted using the difference in HIV annual transmission rates between MSM with HIV who knew their status and were not virologically suppressed and MSM with HIV who did not know their status.

Results: We enrolled 297 participants of whom 150 received MSM peer HIVST kit distribution (intervention group) and 147 received TASO standard of care HIV testing (control group). Provider cost for the intervention was $\$ 2,276$ compared with $\$ 1,827$ for SOC during the 3-month study period. Overall, the intervention resulted in higher HIV positivity yield (4.9 vs. 1.4\%) and averted more HIV infections per quarter (0.364 vs. $0.104)$ compared with SOC. The cost per person tested was higher for the intervention compared to SOC (\$15.90 vs. \$12.40). Importantly, the cost per new HIV diagnosis (\$325 vs. \$914) and cost per transmission averted $(\$ 6,253$ vs. $\$ 17,567)$ were lower for the intervention approach relative to SOC. The incremental cost per HIV transmission averted by the self-testing program was $\$ 1,727$. The incremental cost to providers per additional HIV-positive person identified by the intervention was $\$ 147.30$.

Conclusion: The intervention strategy was cost-effective, and identified more undiagnosed HIV infections than SOC hotspot testing at a cost-effectiveness threshold of US $\$ 2,129$. Secondary distribution of HIVST kits through peers should further be evaluated with longer duration aimed at diagnosing $95 \%$ of all persons with HIV by 2030; the first UNAIDS 95-95-95 target.

Keywords: MSM, HIV, self-testing, peers, cost-effectiveness, Uganda 


\section{INTRODUCTION}

Key populations in Uganda, including sex workers, fisher folk, prisoners, and Men having sex with Men (MSM), are disproportionately affected by HIV and account for more than a third of new HIV infections $(1,2)$. The risk of HIV acquisition is estimated to be 28 times higher among MSM than heterosexual men (3). In 2012, HIV prevalence among MSM (13.2\%) (3) was thrice that of heterosexual adult men aged 15-49 years (4.3\%) in Uganda (4). A mathematical model suggests that the biggest reductions in HIV incidence in SubSaharan Africa will occur through increased coverage of HIV testing and effective treatment of people living with HIV (5). In Uganda, HIV testing uptake among men is low (55\%) compared to $82 \%$ among women (6). No data are available regarding HIV testing coverage among MSM in Uganda where same sex relationships are criminalized through colonial-era laws $(7,8)$. The Anti-Homosexuality Act was passed in 2014 but subsequently overturned by the Constitutional Court of Uganda $(7,8)$. However, social and healthcare stigma and discrimination still hamper key population access to HIV prevention services (6) despite the fact that the Uganda Ministry of Health prohibits discrimination of key populations $(7,8)$.

Scaling up cost effective strategies for HIV testing and counseling services is paramount in order to effectively reach individuals unaware of their HIV status and/or embedded in risky sexual networks. HIV self-testing (HIVST) is the process by which a person performs an HIV test by themselves to know their HIV sero-status (9). OraQuick is approved by the Ministry of Health, and HIVST is recommended in national guidelines as an additional approach to HIV testing services (9). It is an accessible prevention tool that can empower MSM to overcome stigma and discrimination and increase access to HIV testing. Delivering HIVST through peer and sexual networks (9) could be synergistic to existing MSM HIV prevention programmes by reaching MSM, a high-burden population with limited access to HIV testing services, with user-friendly technology (10-13). Prior studies suggest that most MSM would be willing to distribute HIVST kits as well as to self-test in the presence of a peer or sexual partner $(9,14-18)$.

An internet peer MSM HIVST kit distribution strategy in the United states averted 3.34 HIV transmissions among 1,325 MSM over 12 months and saved 14.86 QALYs and \$1.6 million in lifetime HIV treatment costs with an incremental cost effectiveness ratio (ICER) of $\$ 63,400$ for cost-effectiveness at $\$ 100,000$ cost per QALY threshold (10). To our knowledge, no prior study in sub-Saharan Africa has estimated the costeffectiveness of peer distributed HIV oral fluid self-test kits in MSM sexual and social networks. Understanding cost effectiveness of HIVST kits peer distribution is important to inform programmes and policy makers as HIVST is scaled up. This study aimed to estimate the cost per person tested, incremental cost per HIV infection averted, and cost effectiveness of MSM peer distribution of HIVST kits in Uganda.

\section{METHODS}

\section{Study Design}

We conducted a cost effectiveness analysis of a non-randomized study using a provider perspective (9). We compared the cost-effectiveness of an intervention consisting of MSM peer HIVST kit distribution strategy in identifying undiagnosed HIV infection with the standard of care (SOC) HIV testing approach (hotspot HIV testing) used at The AIDS Support Organization (TASO).

\section{Study Setting}

TASO is the largest and oldest indigenous non-governmental HIV care provider in sub-Saharan Africa. It was founded in 1987 by a group of people living with, or deeply affected, by HIV/AIDS in order to provide psychosocial support and basic medical care to people living with HIV and AIDS. TASO Entebbe and Masaka are two of the 11 TASO HIV care centers of excellence located in Central region of Uganda. By June 2018, TASO Entebbe and Masaka had active client populations of $>6,000$ and $>8,000$, respectively. The study was conducted in two urban sites, located in Entebbe and Masaka, in Central Uganda.

\section{Description of HIV Testing Strategies}

The TASO SOC HIV testing models included hotspot HIV testing and counseling for key populations, and highly stigmatized persons (9). From January-March 2018, TASO healthcare workers performed hotspot HIV testing fortnightly at MSM hotspots identified in partnership with MSM civil society organizations; MSM were mobilized for HIV testing through social networks, social media and word-of-mouth. They were eligible to receive an HIV test if they were: (i) aged $>18$ years or older, and (ii) a member of the identified hotspot. For the intervention, we identified 15 MSM peers, eight in Entebbe and seven in Masaka. Each peer (a person with or without HIV and trusted by MSM community) received 10 serialized HIVST kits (Oraquick ${ }^{\circledR}$ Rapid HIV-1/2 Antibody Test, Orasure Technologies, Bethlehem, PA) to distribute to individuals (henceforth referred as participants) in their social and sexual networks who had not tested in the previous 6 months. OraQuick ${ }^{\circledR}$ is a U.S. Food and Drug Administration and Uganda Ministry of Health approved in-home test for HIV-1 and HIV2 that uses oral fluids. The kit consists of a test swab to collect oral fluid from the user's gums, which is then placed in buffered developer solution and results read after $20-40 \mathrm{~min}$. Peers trained the participants on how to use the HIVST kit and interpret the results. Peers provided pre- and post-test HIV counseling, followed up participants through phone calls and face-to-face meetings, collected used kits, and linked those who tested positive to a blood-based confirmatory HIV testing and ART initiation as previously reported (9) (Table 1). To be eligible for MSM peer HIVST kit distribution, participants: (i) were identified by peers, (ii) aged 18 years or older, (iii) had receptive or insertive anal sex with men in the past year.

\section{Cost Data Collection and Analysis}

We used an ingredients approach to estimate the cost of MSM peer HIVST kit distribution and hotspot SOC approaches 
TABLE 1 | Comparison of MSM peer HIVST kits distribution (intervention) and hotspot HIV testing (standard of care) at the AIDS support organization in Uganda.

\begin{tabular}{|c|c|c|}
\hline Variable & $\begin{array}{l}\text { Standard of care } \\
\text { hotspot HIV testing }\end{array}$ & $\begin{array}{l}\text { Intervention (Peer } \\
\text { HIVST distribution) }\end{array}$ \\
\hline Type of test kit & $\begin{array}{l}\text { Blood based HIV rapid } \\
\text { test kits (Determine }{ }^{\circledR} \\
\text { and Uni-Gold }{ }^{\circledR} \text { ) }\end{array}$ & $\begin{array}{l}\text { Oral fluid based } \\
\text { OraQuick }{ }^{\circledR} \text { HIV self-test } \\
\text { kit }\end{array}$ \\
\hline $\begin{array}{l}\text { Mobilization of } \\
\text { participants }\end{array}$ & $\begin{array}{l}\text { Healthcare workers } \\
\text { and drop in center } \\
\text { leadership }\end{array}$ & $\begin{array}{l}\text { Peers (MSM either HIV } \\
\text { infected or not, } \\
\text { identified by MSM } \\
\text { community) }\end{array}$ \\
\hline Performer & Healthcare worker & $\begin{array}{l}\text { Self or peer assisted or } \\
\text { HIVST }\end{array}$ \\
\hline Where & $\begin{array}{l}\text { Hotspots where MSM } \\
\text { meet }\end{array}$ & $\begin{array}{l}\text { Place of participant's } \\
\text { choice }\end{array}$ \\
\hline Linkage to care & $\begin{array}{l}\text { Referral letters given by } \\
\text { the healthcare workers }\end{array}$ & $\begin{array}{l}\text { Peers used phone } \\
\text { calls, face to face } \\
\text { meeting including } \\
\text { physically linking MSM } \\
\text { to confirmatory testing } \\
\text { and care }\end{array}$ \\
\hline HIV counseling & Healthcare worker & $\begin{array}{l}\text { Peers (both } \\
\text { HIV-positive and } \\
\text { negative MSM) }\end{array}$ \\
\hline Sample size & 147 participants & 150 participants \\
\hline $\begin{array}{l}\text { Completed the } \\
\text { test }\end{array}$ & 147 participants & 143 participants \\
\hline Duration & January-March 2018 & June-August 2018 \\
\hline
\end{tabular}

(11). For intervention cost estimation, we retrieved and reviewed 2020 intervention expenditures reported in US dollars which included formative research, administrative, overhead costs, and intervention implementation costs from financial records and reports. We extracted research project expenditure including both costs for formative research and peer HIVST kit distribution. Thereafter, we identified MSM peer HIVST kit distribution (intervention) ingredients, estimated costs likely to be provider costs using the expenditure report, and excluded formative research costs. We categorized costs as fixed costs that remained unchanged over the short-term regardless of the number of participants, and variable costs likely to increase or decrease according to the number of participants. Fixed costs included personnel, training, and administration. Variable costs were direct provider costs including HIV test kits, monitoring of peer HIVST kit distribution, and costs of participant tracing and peer stipends. Data collection for the SOC group (JanuaryMarch 2018) was not synchronous with the intervention group (June-August 2018) (Table 2).

\section{Standard of Care Costing Estimation}

We reviewed TASO key population programme data and reports between January and March 2018 to identify the number of persons tested during hotspot campaigns as previously described (9). Each month, TASO staff conducted a maximum of two hotspot testing sessions. A total of nine hotspot-testing activities were conducted during the study period, five in Entebbe and four in Masaka. We interviewed TASO staff to estimate time
TABLE 2 | Estimated costs (US dollars) of the intervention (MSM peer HIVST distribution) and standard of care.

\begin{tabular}{|c|c|c|}
\hline Programme activities & $\begin{array}{c}\text { Intervention } \\
\text { Peer HIVST } \\
\text { distribution provider } \\
\text { cost (\$) }\end{array}$ & $\begin{array}{c}\text { Standard of care } \\
\text { Hotspot HIV testin } \\
\text { provider cost }(\$)\end{array}$ \\
\hline \multicolumn{3}{|l|}{ Programme start-up costs } \\
\hline MSM peer identification and training & 148.90 & $\mathrm{~N} / \mathrm{A}$ \\
\hline Venue identification and facilitation & N/A & 82.80 \\
\hline \multicolumn{3}{|l|}{ Personnel and Administration cost } \\
\hline Project coordinator & 449.30 & $\mathrm{~N} / \mathrm{A}$ \\
\hline Counselor coordinator & N/A & 720 \\
\hline Laboratory technician & N/A & 619.20 \\
\hline Research assistants & 168 & $\mathrm{~N} / \mathrm{A}$ \\
\hline Data manager & 151 & N/A \\
\hline \multicolumn{3}{|l|}{ Variable costs } \\
\hline Transport & 250 & 46 \\
\hline Mobilization of MSM to hotspots & N/A & 155 \\
\hline HIV testing & $1,008.80$ & 204 \\
\hline Follow up and reporting & 99.0 & N/A \\
\hline Total cost & $\$ 2,276$ & $\$ 1,827$ \\
\hline
\end{tabular}

The counselor at TASO also works as a Coordinator. The laboratory technician performs HIV testing, manages the data and enters it into the HIV testing register. MSM peers provided pre- and post-test HIV counseling including linkage to a confirmatory test and ART initiation. Transport costs, is for peer transport reimbursement (stipend) for the intervention arm and TASO staff transport to the hotspot for the control arm. HIV testing, is cost for purchasing HIVST kits for the interventional arm and control arm.

(in hours) spent during hotspot testing. We identified ingredient activities to identify provider costs of hotspot testing. We used the 2018 public sector cost of $\$ 1.02$ to estimate the total cost of Determine $^{\circledR}$ HIV1/2 rapid test kits (Alere Medical Company, Chiba, Japan).

For both groups, we estimated: (a) the cost per person tested by dividing total costs by number of HIV tests completed, (b) the cost per new HIV diagnosis by dividing total costs by the number of new (not diagnosed before) HIV infections identified, and (c) the cost per transmission averted by dividing total programme cost by the number of HIV infections averted.

\section{Cost-Effectiveness Analysis}

Cost effectiveness was defined as the number of HIV transmissions averted using a Bernoulli model to estimate averted transmissions among MSM (12). The number of transmissions averted was estimated using the difference in HIV annual transmission rate between HIV-positive MSM who knew their status and were not virologically suppressed (6.9\%) and HIV-positive MSM who did not know their status (12.1\%) (12). MSM who do not know their HIV status transmit HIV infection at a higher annual rate than those who know their HIV status (12). Since all MSM who completed an HIV test in both groups received their test results and were initiated on treatment, we assumed that the HIV transmission rate dropped after HIV diagnosis and immediate initiation of treatment. We therefore estimated the number of HIV transmissions averted by 
multiplying the number of new diagnoses by the difference in HIV transmission rates before and after HIV diagnosis. This was calculated for each HIV testing approach using the formula $\mathrm{a}=$ $\mathrm{Nu}(\mathrm{Tu}-\mathrm{Ta})$ where $\mathrm{a}$ is the number of averted HIV transmissions, $\mathrm{Nu}$ is undiagnosed HIV infections, $\mathrm{Tu}$ is the average HIV transmission rate from MSM unaware of their status, and Ta is the average HIV transmission rate from MSM aware of their status (13).

The cost-effectiveness threshold was set at US \$2,130, following the World Health Organization (WHO) "CHOosing Interventions that are Cost-Effective (CHOICE)" recommended threshold for cost effectiveness analysis, i.e., thrice the Uganda gross domestic product per capital of US $\$ 710$ in 2018 (18-20). We used WHO threshold because we found no comparable HIV prevention (HIV testing) study estimating QALY gained or DALY averted among MSM in a similar setting and did not collect quality of life data. We calculated the incremental cost effectiveness ratio (ICER), defined as $\Delta \mathrm{C} / \Delta \mathrm{E}=\mathrm{Cb}-\mathrm{Ca} / \mathrm{Eb}-\mathrm{Ea}$ where $\mathrm{C}$ is total programme cost, $\mathbf{E}$ is effectiveness (averted infections), $\mathbf{b}$ is the control index, and $\mathbf{a}$ is the intervention.

\section{Sensitivity Analysis}

We tested the robustness of the intervention cost effectiveness analysis by using a weighted average transmission rate half $(6.9 \% / 2=3.45 \%)$ the transmission rate for MSM aware of their HIV-positive status and not virologically suppressed (12), taking into consideration participants who completed an HIV test, diagnosed HIV- positive and initiated on ART with good peer adherence support system to achieve viral suppression. For our sensitivity analysis, we assumed that newly diagnosed MSM in the intervention group were more likely to be linked to care by peers and initiate ART than those in the SOC group because the intervention participants received HIV counselling and prevention messaging from a peer who is familiar with them. Thus, we halved the proportion of MSM who engaged in risky behaviours in the intervention group. We also added the cost of confirmatory HIV testing ( $\$ 1.02$ per Determine ${ }^{\circledR}$ rapid test and $\$ 3.40$ per Uni-Gold ${ }^{\circledR}$ rapid test (2018 market price) for the eight participants diagnosed with HIV infection using HIVST. We also included personnel costs of confirmatory testing, assuming $\sim 30 \mathrm{~min}$ of HIV testing and counseling were equivalent to $3.4 \%$ effort (mean monthly salary of $\$ 800$ per TASO provider).

\section{Ethics Approval}

This study was approved by the Infectious Diseases Institute Scientific Review Committee, TASO Research Ethics Committee, The University of California, San Francisco Ethics Committee, and the Uganda National Council for Science and Technology (UNCST). The English or Luganda (local language) information sheet and the verbal informed consent tool approved by the TASO Research Ethics Committee and UNCST was explained to the participant. Those who agreed to take part in the study provided verbal consent that was not documented, consistent with guidelines from regulatory bodies concerned about the criminalization of MSM in Uganda (21).
TABLE 3 | Cost effectiveness of MSM peer HIVST kit distribution and hotspot HIV testing in Uganda.

\begin{tabular}{|c|c|c|}
\hline Measure & $\begin{array}{c}\text { MSM peer HIVST kits } \\
\text { distribution }\end{array}$ & $\begin{array}{c}\text { Hotspot HIV } \\
\text { testing }\end{array}$ \\
\hline a. Number of tests ${ }^{a}$ & 143 & 147 \\
\hline b. Total number testing positive & 8 & 4 \\
\hline c. Number testing positive, aware & 1 & 2 \\
\hline d. Number testing positive, unaware & 7 & 2 \\
\hline $\begin{array}{l}\text { e. Proportion testing } \\
\text { positive, unaware }\end{array}$ & 0.049 & 0.014 \\
\hline $\begin{array}{l}\text { f. Transmission rate from MSM } \\
\text { unaware HIV+ }\end{array}$ & 0.121 & 0.121 \\
\hline $\begin{array}{l}\text { g. Transmission rate from MSM } \\
\text { aware HIV+ }\end{array}$ & 0.069 & 0.069 \\
\hline h. Number of infections averted ${ }^{b, c}$ & 0.364 & 0.104 \\
\hline i. Total provider costs $(\$)$ & 2,276 & 1,827 \\
\hline j. Cost per person tested & 15.90 & 12.40 \\
\hline k. Cost per new diagnosis $(\$)$ & 325 & 914 \\
\hline I. Cost per averted infection (\$) & 6,253 & 17,567 \\
\hline $\begin{array}{l}\text { Incremental CE ratio (ICER), per } \\
\text { averted infection }\end{array}$ & $\$ 1,727$ & \\
\hline
\end{tabular}

a Market price of 2018 per Determine HIV test (\$1.02) was used to estimate the cost of hotspot testing.

${ }^{b}$ The number of averted HIV infections was estimated by multiplying the number of MSM with HIV who became aware of their status and the difference in transmission rates before and after knowing their HIV status. $h=d^{*}[f-g]$.

${ }^{c}$ The average HIV transmission rate for all groups was used for the number of averted infections.

\section{RESULTS}

Overall, 297 participants were included in the analysis of which 150 received HIVST (intervention) and 147 were reached with SOC HIV testing during the 3-month study period as previously described (9). A total of 143 participants (95\%) completed HIVST, of whom $32 \%$ had never tested for HIV. All participants in the control group (100\%) received SOC testing. Overall, a total of 12 participants were diagnosed with HIV infection: eight in the intervention group and four in the SOC group [5.6 vs. $2.7 \%$, respectively; $P=0.02$ ]. All participants newly diagnosed HIV-positive using HIVST received confirmatory $\mathrm{HIV}$ testing, were linked to care by the peers and initiated on treatment. Details about SOC and HIVST.

The total provider cost for MSM peer HIVST distribution (intervention) was $\$ 2,276$ compared with $\$ 1,827$ for hotspot testing (SOC). Using the HIV transmission rate averted between MSM of known status but not virally suppressed, and unknown HIV status, the intervention resulted in a higher HIV positivity rate (4.9 vs. $1.4 \%$ ) vs. SOC during 3 months of implementation. Compared to the control group, the intervention strategy averted more HIV transmissions per quarter (0.364 vs. 0.104) but yielded a higher cost per person tested (\$15.90 vs. \$12.40). The cost per new HIV diagnosis (\$914 vs. \$325) and the cost per HIV transmission $(\$ 17,567$ vs. $\$ 6,253)$ averted were higher for the SOC than the intervention. The incremental cost per 
transmission averted by the self-testing program was $\$ 1,727$ (Table 3).

In sensitivity analysis, adding the cost of confirmatory costing increased the cost per person tested from $\$ 15.90$ to $\$ 16.50$ and halving the the transmission rate for MSM aware of their HIV-positive status and not virologically suppressed increased the number of HIV infections averted from 0.364 to 0.602 . The cost per infection averted reduced from $\$ 6,253$ to $\$ 3,914$. MSM peer HIVST distribution remained cost-effective (ICER $\$ 1,062$ ) in identifying new infections and the incremental cost per transmission averted by the self-testing program remained cost effective (Table 4).

The incremental cost to providers per additional HIV-positive person identified by the intervention was $\$ 147.30$ (Table 5).

\section{DISCUSSION}

To our knowledge, this is first study to examine the costeffectiveness of peer HIVST distribution in sub-Saharan Africa. Our provider perspective analysis found that the MSM peer HIVST kit distribution strategy was cost-effective than SOC hotspot testing in identifying undiagnosed HIV infections among MSM who are a hidden, highly stigmatized population in Uganda and hard to reach with HIV services. The average cost per person tested through MSM peer HIVST kit distribution was higher than hotspot testing because of the higher cost of the Oraquick ${ }^{\circledR}$ HIVST kit relative to the Determine ${ }^{\circledR}$ HIV rapid kit ( $\$ 6.72$ vs. \$1.02), respectively. The MSM peer HIVST kits distribution strategy averted thrice as many HIV infections as hotspot testing, potentially lowering the risk of HIV transmission from MSM unaware of their HIV status.

Previous studies have found that HIVST distribution strategies are cost-effective for heterosexual populations in sub Saharan Africa $(22,23)$. Our findings are in agreement with an internet MSM peer HIVST kits distribution strategy that reached 1,325 MSM over a 12 month period and found that peer based HIVST kit distribution was cost-effective in the United States (10). Furthermore, a modeling study of HIV testing interventions that included lifetime treatment, quality-adjusted life years and 12 months of implementation still found that HIVST delivered through social and sexual networks remained cost effective for identifying undiagnosed HIV infections (17). Reaching high-risk MSM with HIV testing services is key to facilitating early diagnosis of HIV infection and linkage to HIV services. Immediate initiation of antiretroviral therapy has personal health benefits (decreased morbidity and mortality) and public health benefits (prevention of sexual transmission of HIV) (24). MSM peer HIVST kit distribution could address

TABLE 4 | Cost effectiveness of MSM peer HIVST kit distribution and hotspot HIV testing for different HIV transmission rates by type of testing.

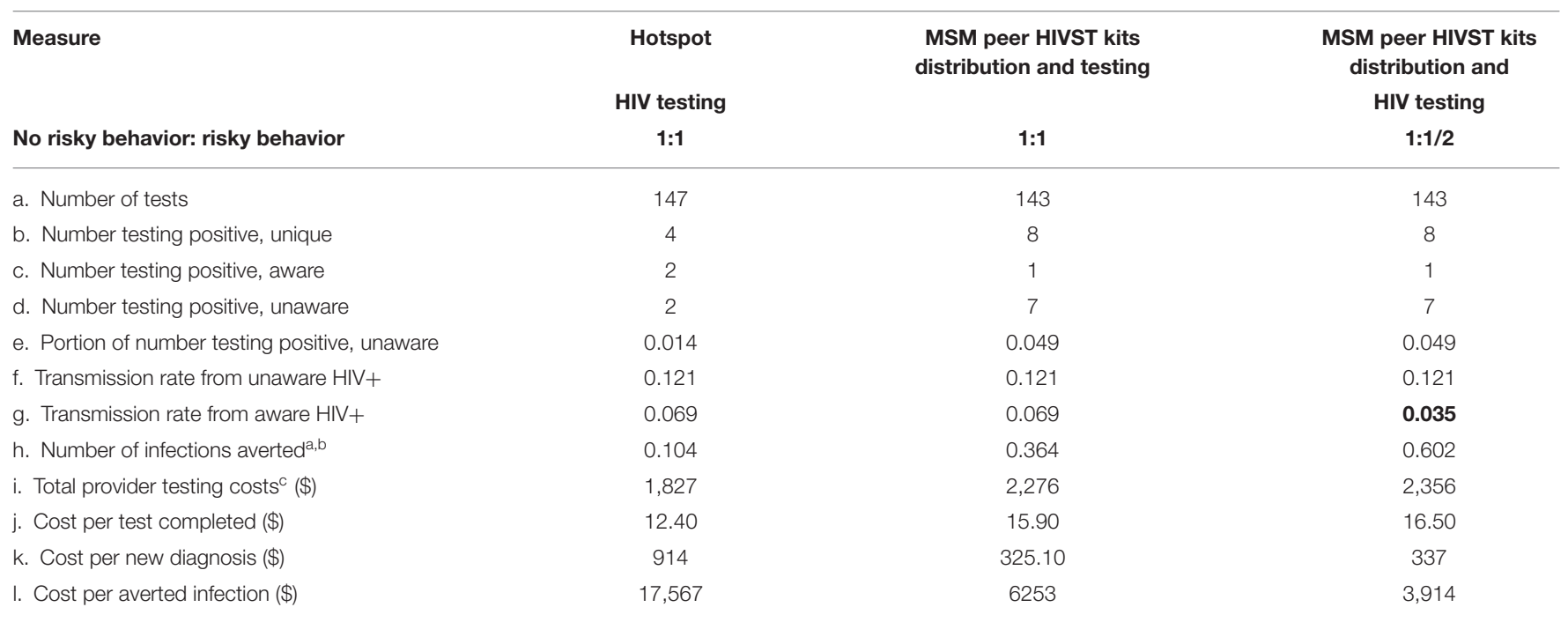

a The number of averted HIV infections was estimated by multiplying the number of MSM with HIV who became aware of their status and the difference in transmission rates before and after knowing their HIV status. $h=d^{*}[f-g]$.

${ }^{b}$ The average HIV transmission rate for all groups was used for the number of averted transmissions.

${ }^{c}$ We added cost of confirmatory testing using rapid test kit market rate of 2018 and provider cost of counseling and testing.

TABLE 5 | Providers incremental cost for the peer HIVST distribution strategy.

\begin{tabular}{lcccc}
\hline Strategy & Provider costs & Incremental cost \$ & $\begin{array}{c}\text { Effectiveness (HIV } \\
\text { positive diagnosed) }\end{array}$ & $\begin{array}{c}\text { Incremental } \\
\text { effectiveness (+ve) }\end{array}$ \\
\hline Standard of care arm & 1,827 & - & 4 & - \\
Intervention arm & 2,276 & 449 & 8 & 4
\end{tabular}


gaps in HIV testing services by increasing testing coverage and frequency and increasing the proportion of first time testers and new HIV diagnoses in stigmatized high-risk populations such as MSM and sex workers in sub-Saharan Africa $(15,25,26)$.

The cost per person tested in our study ( $\$ 15.90$ and $\$ 12.40$ for peer distribution and hotspot testing, respectively) compares favorably with prior studies in sub-Saharan Africa in which the average cost per person tested using door to door community based HIVST distribution was $\$ 13.00$ (range, \$8.78-\$16.42): $\$ 8.78$ in Malawi, $\$ 16.42$ in Zambia, and \$13.84 in Zimbabwe (22). Door-to-door and peer HIVST distribution are communitybased HIV testing strategies with similar costs per person tested, but only the latter approach is suitable for hidden populations like MSM. The cost per HIV kit distributed in our peer distribution programme would significantly decrease if it were integrated within the established TASO key population HIV prevention programme in which staff and peers mobilize MSM for HIV testing at hotspots and link them to HIV services. Integration would reduce personnel costs and increase the number of MSM reached with HIVST. In the FHI 360 linkage project (23), MSM peers integrated within the HIV testing programme distributed over 500 kits within 3 months, indicating that peers can efficiently distribute HIVST kits, potentially decreasing the cost per HIVST kit distributed and increasing the yield of persons testing HIV-positive. The incremental provider cost per additional HIV positive person identified by MSM peer HIVST kits distribution was $\$ 147.30$ (Table 5). This additional cost is considered high given that it is almost thrice Uganda national health expenditure on health per capital - spending of \$55 in 2017 (27). However, our current study findings show that the MSM peer HIVST kit distribution approach is cost effective in identifying HIV infection in this stigmatized population.

Scale-up of MSM peer based HIV testing approaches in sub-Saharan Africa in general, and in Uganda in particular, is hampered by criminalization of homosexual behavior and scarcity of evidence on the cost-effectiveness of peer distribution programmes $(7,8)$. Our results suggest that HIVST can efficiently reach a high-risk marginalized population in need of HIV services. The President's Emergency Plan for AIDS Relief (PEPFAR) is working with the Ministry of Health and HIV implementing partners in Uganda through the local capacity initiative to strengthen the national capacity of key population civil society organizations to address barriers to HIV care, support and prevention services among the MSM community and to understand how best to reach them with HIV services (28). Our results will inform HIV programmes and policy makers on key considerations when scaling up peer distribution of HIVST kits to MSM social and sexual networks. In Uganda, peer distribution of HIVST kits is being scaled up for key populations including MSM and male and female sex workers (29-31). Our findings support the cost-effectiveness of this approach. However, there is need to evaluate the cost-effectiveness of HIVST differentiated delivery models for other key populations and the cost-effectiveness of frequent testing for MSM as recommended by WHO. Quality assurance of HIVST kits is needed since products of unknown quality are available on the unregulated market, with attendant risks of false positive/negative results, and underscoring the need to strengthen consumer protections $(25,32)$.

Sensitivity analyses including the cost of confirmatory testing and a different set of assumptions for differences in transmission risk found that the cost per person tested increased marginally from $\$ 15.90$ to $\$ 16.50$. Importantly, the number of infections averted were increased from $36.4 \%$ to $60.2 \%$ and the cost per infection averted was halved from $\$ 6,253$ to $\$ 3,914$. MSM peer HIVST kit distribution remained cost effective in our study (ICER \$1,062) (Table 4).

A strength of our study is the first cost-effectiveness evaluation of peer-distributed HIVST for MSM in sub-Saharan Africa. Our study has limitations. Study duration was only 3 months and mostly reached younger MSM in a setting where older MSM are harder to reach but have higher HIV prevalence. However, younger MSM are at higher risk of HIV acquisition despite lower HIV prevalence; risk of HIV infection increases with age (3). The number of HIV self-tests distributed was relatively small and the comparator was hotspot moonlight testing and not facility-based HIV testing services, thus limiting generalizability of our results. We relied on self-report of prior HIV status and some participants may incorrectly have reported their status. MSM peer HIVST kit distribution (June-August 2018) was not synchronous with programmatic SOC testing (January-March 2018); nevertheless, the 3-month offset did not influence cost estimates. The cost analysis was not specified a priori and relied on data from study implementation costs reported to the funder. Initial expenditures included costs of formative research and we may have under- or overestimated implementation costs. We used a provider perspective that excludes patient costs, which are a key barrier to accessing HIV testing services in Uganda. However, in our intervention, patient costs should have been minimal since HIVST kits were distributed to participants at their locations of preference. Finally, we used transmission risk data from the USA; HIV transmission risk among MSM in Uganda is unknown and is likely different (higher or lower) than the United States.

In conclusion, secondary distribution of HIVST kits by MSM peers was cost-effective and identified more undiagnosed HIV infections than SOC approaches. HIVST peer distribution should further be evaluated with longer durations aimed at underserved and hard-to-reach MSM at risk of HIV infection with the goal of expanding testing coverage to $95 \%$ of all persons with HIV (UNAIDS first 95 target).

\section{DATA AVAILABILITY STATEMENT}

The original contributions presented in the study are included in the article/supplementary material, further inquiries can be directed to the corresponding author/s.

\section{ETHICS STATEMENT}

The studies involving human participants were reviewed and approved by this study was approved The Infectious 
Diseases Institute Scientific Review Committee, TASO Research Ethics Committee, The University of California, San Francisco Ethics Committee, and the Uganda National Council for Science and Technology (UNCST). The English or Luganda (local language) information sheet and the verbal informed consent tool approved by the TASO Research Ethics Committee and UNCST was explained to the participant. Those who agreed to take part in the study provided verbal consent that was not documented, consistent with guidelines from regulatory bodies concerned about the criminalization of MSM in Uganda. Written informed consent for participation was not required for this study in accordance with the national legislation and the institutional requirements.

\section{AUTHOR CONTRIBUTIONS}

SO designed the study and wrote the first draft along with AM. SO, YK, PO, and BC performed the statistical analyses. All authors contributed to data collection, interpretation of the results and the writing of the manuscript, and approved the final draft.

\section{REFERENCES}

1. Hladik W, Barker J, Ssenkusu JM, Opio A, Tappero JW, Hakim $A$, et al. HIV infection among men who have sex with men in kampala, uganda-a respondent driven sampling survey. PLoS ONE. (2012) 7:e38143. doi: 10.1371/journal.pone.0038143

2. Hladik W, Sande E, Berry M, Ganafa S, Kiyingi H, Kusiima J, et al. Men who have sex with men in Kampala, Uganda: results from a biobehavioral respondent driven sampling survey. AIDS Behav. (2017) 21:147890. doi: 10.1007/s10461-016-1535-2

3. UNAIDS (Joint United Nations Programme on HIV/AIDS). Global AIDS Update (2018) Miles To Go: Closing Gaps Breaking Barriers Righting Injustices. Unaids. Available online at: https://www.unaids.org/sites/default/files/media_ as.

4. Uganda Population Based HIV Impact Assessment, 2016-2017 Report.

5. Kelly SL, Wilson DP. HIV cascade monitoring and simple modeling reveal potential for reductions in HIV incidence. J Acquir Immune Defic Syndr. (2015) 69:257-63. doi: 10.1097/QAI.0000000000000655

6. King R, Sebyala Z, Ogwal M, Aluzimbi G, Apondi R, Reynolds $S$, et al. How men who have sex with men experience HIV health services in Kampala, Uganda. BMJ Global Healt. (2020) 5:e001901. doi: 10.1136/bmjgh-2019-001901

7. Uganda Penal Code Act, Chapter 120, Section 145. Laws of Uganda. (1950). Available online at: https://ulii.org/ug/legislation/consolidated-act/ 120 (accessed December 4th, 2020).

8. Oloka-Onyango \& 9 Others vs. Attorney General. Constitutional Petition no. 08 of 2014. Available online at: http://www.ulii.org/ug/judgment/ constitutional-court/2014/14/ (accessed May 11, 2020).

9. Okoboi S, Lazarus O, Castelnuovo B, Nanfuka M, Kambugu A, Mujugira A, et al. Peer distribution of HIV self-test kits to men who have sex with men to identify undiagnosed HIV infection in Uganda: a pilot study. PLoS ONE. (2020) 15:e0227741. doi: 10.1371/journal.pone.0227741

10. Shrestha RK, Chavez PR, Noble M, Sansom SL, Sullivan PS, Mermin $\mathrm{JH}$, et al. Estimating the costs and cost-effectiveness of HIV self-testing among men who have sex with men, United States. J Int AIDS Soc. (2020) 23:e25445. doi: 10.1002/jia2.25445

\section{FUNDING}

This study was supported through funding from University of California Centre for AIDS Research (UCSF CFAR International Mentored Scientist Award, grant P30-AI027763 to SO) and Fogarty International Center, National Institutes of Health (grant \# 2D43TW009771-06 "HIV and co-infections in Uganda". AM was supported by the Fogarty International Center of the US National Institutes of Health (grant K43 TW010695). The authors report no conflicts of interest. The contents are solely the views of the authors and do not necessarily represent those of the funding organizations.

\section{ACKNOWLEDGMENTS}

We gratefully acknowledge the contributions of study participants, peers and TASO leadership at Entebbe and Masaka HIV care centers for allowing us to conduct this pilot study within their regular MSM programming. We are indebted to the TASO Program Director, Dr. Kenneth Mugisha, and the Executive Director of TASO, Dr. Bernard Michael Etukoit for administratively supporting this study and to the participants for taking part in the study.

11. Barton P, Bryan S, Robinson S. Modelling in the economic evaluation of health care: Selecting the appropriate approach. J Health Serv Res Policy. (2004) 9:110-8. doi: 10.1258/135581904322987535

12. Lin F, Farnham PG, Shrestha RK, Mermin J, Sansom SL. Cost effectiveness of HIV prevention interventions in the US. Am J Prev Med. (2016) 50:699708. doi: 10.1016/j.amepre.2016.01.011

13. Castel AD, Choi S, Dor A, Skillicorn J, Peterson J, Rocha N, et al. Comparing cost-effectiveness of HIV testing strategies: targeted and routine testing in Washington, DC. PLoS ONE. (2015) 10:1-11. doi: 10.1371/journal.pone.0139605

14. Sharma A, Chavez PR, MacGowan RJ, McNaghten AD, Mustanski B, Gravens L, et al. Willingness to distribute free rapid home HIV test kits and to test with social or sexual network associates among men who have sex with men in the United States. AIDS Care. (2017) 29:1499503. doi: 10.1080/09540121.2017.1313386

15. Lippman SA, Lane T, Rabede O, Gilmore H, Chen YH, Mlotshwa N, et al. High acceptability and increased HIV-testing frequency after introduction of HIV self-testing and network distribution among South African MSM. J Acquir Immune Defic Syndr. (2018) 77:279-87. doi: 10.1097/QAI.000000000000 1601

16. Lippman SA, Lane T, Rabede O, Gilmore H, Chen YH, Mlotshwa N, et al. HIV self-test distribution increases test frequency in South African MSM. Top Antivir Med. (2018) 26:63s-64s.

17. Hutchinson AB, Farnham PG, Sansom SL, Yaylali E, Mermin JH. Cost-effectiveness of frequent HIV testing of high-risk populations in the United States. J Acquir Immune Defic Syndr. (2016) 73:323-30. doi: 10.1097/QAI.0000000000000838

18. Woods B, Revill P, Sculpher M, Claxton K. Country-level cost-effectiveness thresholds: initial estimates and the need for further research. Value Health. (2016) 19:929-35. doi: 10.1016/j.jval.2016.02.017

19. Marseille E, Larson B, Kazi DS, Kahn JG, Rosen S. Thresholds for the cost-effectiveness of interventions: alternative approaches. Bull World Health Organ. (2015) 93:118-24. doi: 10.2471/BLT.14.138206

20. Uganda GDP Growth (annual). Budgeting and Public Expenditures in OECD Countries. Kampala: Uganda National Council for Science and Technology (2019). 
21. Uganda National Council for Science \& Technology. National Guidelines for Research Involving Humans as Research participants. (2014).

22. Mangenah C, Mwenge L, Sande L, Ahmed N, d'Elbée M, Chiwawa P, et al. Economic cost analysis of door-to-door community-based distribution of HIV self-test kits in Malawi, Zambia, and Zimbabwe. J Int AIDS Soc. (2019) 22:e25255. doi: 10.1002/jia2.25255

23. FHI360 Linkage Project. (2020). Available online at:https://www.fhi360. org/sites/default/files/media/documents/resource-linkages-burundiachievements.pdf. (accessed April 20, 2020).

24. Cohen MS, Chen YQ, McCauley M, Gamble T, Hosseinipour MC, Kumarasamy N, et al. Antiretroviral therapy for the prevention of HIV-1 transmission. N Engl J Med. (2016) 375:830-9. doi: 10.1056/NEJMoa1600693

25. Thirumurthy $\mathrm{H}$, Masters SH, Mavedzenge SN, Maman S, Omanga E, Agot K. Promoting male partner HIV testing and safer sexual decision making through secondary distribution of self-tests by HIVnegative female sex workers and women receiving antenatal and postpartum care in Kenya: a cohort study. Lancet HIV. (2016) 3:e26674. doi: 10.1016/S2352-3018(16)00041-2

26. Sharma M, Ying R, Tarr G, Barnabas R. Systematic review and meta-analysis of community and facility-based HIV testing to address linkage to care gaps in sub-Saharan Africa. Nature. (2015) 528:S77-85. doi: 10.1038/nature16044

27. Uganda National Health Expenditure, 2017 Report.

28. Fact Sheet. PEPFAR Strategy for Accelerating HIV/AIDS Epidemic Control (2017-2020). Kampala: PEPFAR (2017). p. 18.
29. Uganda Ministry of Health. Distribution of HIV Self Test Kits to Public Health Facilities. Geneva: WHO (2018).

30. WHO Policy Brief. Consolidated Guidelines on HIV Testing Services for a Changing Epidemic. Geneva: WHO (2019).

31. WHO Policy Brief. Who recommends social network-based HIV testing approaches for key populations as part of partner services package. JAMA. (2014) 312:1-8.

32. MacPherson P, Lalloo DG, Webb EL, Maheswaran H, Choko AT, Makombe SD, et al. Effect of optional home initiation of HIV care following HIV self-testing on antiretroviral therapy initiation among adults in Malawi: a randomized clinical trial. JAMA. (2014) 312:3729. doi: 10.1001/jama.2014.6493

Conflict of Interest: The authors declare that the research was conducted in the absence of any commercial or financial relationships that could be construed as a potential conflict of interest.

Copyright () 2021 Okoboi, Castelnuovo, Van Geertruyden, Lazarus, Vu, Kalibala, Kamara, Ochanda, King and Mujugira. This is an open-access article distributed under the terms of the Creative Commons Attribution License (CC BY). The use, distribution or reproduction in other forums is permitted, provided the original author(s) and the copyright owner(s) are credited and that the original publication in this journal is cited, in accordance with accepted academic practice. No use, distribution or reproduction is permitted which does not comply with these terms. 